\title{
Geometrical Impact on Guided Mode Excitation in Solar Cells
}

\author{
Ali Naqavi ${ }^{1,2}$, Franz-Josef Haug ${ }^{1}$, Karin Söderström ${ }^{1}$, Olindo Isabella $^{3}$, Hans Peter Herzig ${ }^{2}$, Christophe Ballif ${ }^{1}$ \\ and Miro Zeman ${ }^{3}$ \\ ${ }^{1}$ Photovoltaics and Thin Film Electronics Laboratory, École Polytechnique Fédérale de Lausanne (EPFL), Breguet 2, 2000 Neuchâtel, \\ Switzerland \\ ${ }^{2}$ Optics \& Photonics Technology Laboratory, École Polytechnique Fédérale de Lausanne (EPFL), Breguet 2, 2000 Neuchâtel, Switzerland \\ ${ }^{3}$ Photovoltaic Materials and Devices/Dimes, Delft University of Technology, Feldmannweg 17, 2628 CT Delft, The Netherlands \\ e-mail: ali.naqavi@epfl.ch
}

\begin{abstract}
We investigate the influence of patterned metallic back reflector on absorption in thinfilm silicon photovoltaics. The impact of symmetry, periodicity, and angle of incidence of light is discussed by simulation and experiment.
\end{abstract}

OCIS codes: $350.6050,040.5350,050.1950,130.2790$.

Achieving high efficiency in thin film photovoltaics requires maximizing the capture of incident photons in the active absorber of the solar cell, as well as an efficient transport of the photogenerated carriers. To increase light absorption, random and periodic textures have been introduced at cell interfaces and/or at backreflector to provide efficient light trapping schemes. Historically, random textures have been interesting due to the ease of fabrication and the high amount of absorption enhancement that they provide. However, periodic textures can theoretically enhance absorption yet more, at least over limited wavelength ranges [1]. Regardless of its shape, interface texturing couples the incident light to the modes of the multilayer stack of the solar cell. The strength of this coupling is determined by the shape of the texture, so, finding textures which excite the guided modes of the solar cell strongly has attracted remarkable interest recently [2-4]. Here, we study the excitation of guided modes in thin film amorphous silicon solar cells based on periodic textures. The results of our analysis might be generalized to other types of solar cells and multilayer structures.

The effect of texture geometry on the absorption enhancement can be investigated in different respects. One basic question is about the symmetry of the texture. It has been predicted that asymmetric patterns can excite more guided modes than their symmetric counterparts $[1,5]$. Generally an asymmetric texture excites more guided modes than a symmetric one but the strength of the coupling to the guided modes is not equal in both cases. It has been reported that thin film solar cell based on asymmetric sawtooth shaped gratings can provide superior photocurrent under normal incidence [6]. Here we show that symmetric patterns can be as good. Furthermore, we investigate the angular response of different textures to consider the impact of incident angle on the absorption.

To address the above questions, we use rigorous coupled wave analysis (RCWA) to simulate a multilayered thin film solar cell with conformal layers based on one-dimensional (1D) metallic gratings shown in Figure 1-a. From top to bottom, the cell includes ITO $(50 \mathrm{~nm})$, p-doped silicon $(15 \mathrm{~nm})$, intrinsic amorphous silicon $(180 \mathrm{~nm})$, n-doped silicon $(20 \mathrm{~nm}), \mathrm{ZnO}(70 \mathrm{~nm})$ and silver as back reflector. The silver is patterned with the depth of $h=300 \mathrm{~nm}$ and period of $P=550 \mathrm{~nm}$. Figure 1 shows the simulated external quantum efficiency (EQE) in P polarization (the electric field parallel to the grating grooves) under different incident angles $(\theta)$ for different grating patterns. The EQE is approximated here as the fraction of incident light which is absorbed in the intrinsic silicon layer. The asymmetric profile does excite more guided modes, but its EQE is not necessarily higher than the one of the symmetric patterns, especially when the dependence of the EQE on the incident angle is considered.

Next we consider the same cell based on a 1D symmetric grating depicted in Figure 2 (a) with the same dimensions as before. Figure 2 (d) shows the EQE of the cell in both polarizations besides the EQE of the cell on a 2D grating which is made by applying the same profile in both $\mathrm{x}$ and $\mathrm{y}$ directions (Figure 2(b)) and is simulated using HFSS package based on 3D finite element method. The EQE is higher in the 2D case than the EQE of both 1D polarizations since the $2 \mathrm{D}$ grating excites more modes compared to the $1 \mathrm{D}$ grating. It is expected that $2 \mathrm{D}$ gratings provide higher EQE than 1D gratings over a wide angular range. Furthermore, because there is a cluster of guided modes which enhances the EQE in 2D gratings, randomly textured cells might behave similarly.

Figure 2 (d) compares the simulated EQE of the previous 2D grating cell with the EQE of an experimental sample based on a $2 \mathrm{D}$ grating with $P=500 \mathrm{~nm}, h=150 \mathrm{~nm}$ and intrinsic Si layer thickness of $250 \mathrm{~nm}$ (Figure 2 (c)). The EQEs agree relatively well to each other after $600 \mathrm{~nm}$. The results reveal that due to the excitation of a multitude of modes, light trapping depends weakly on the geometry of the $2 D$ pattern at long wavelength regime. 

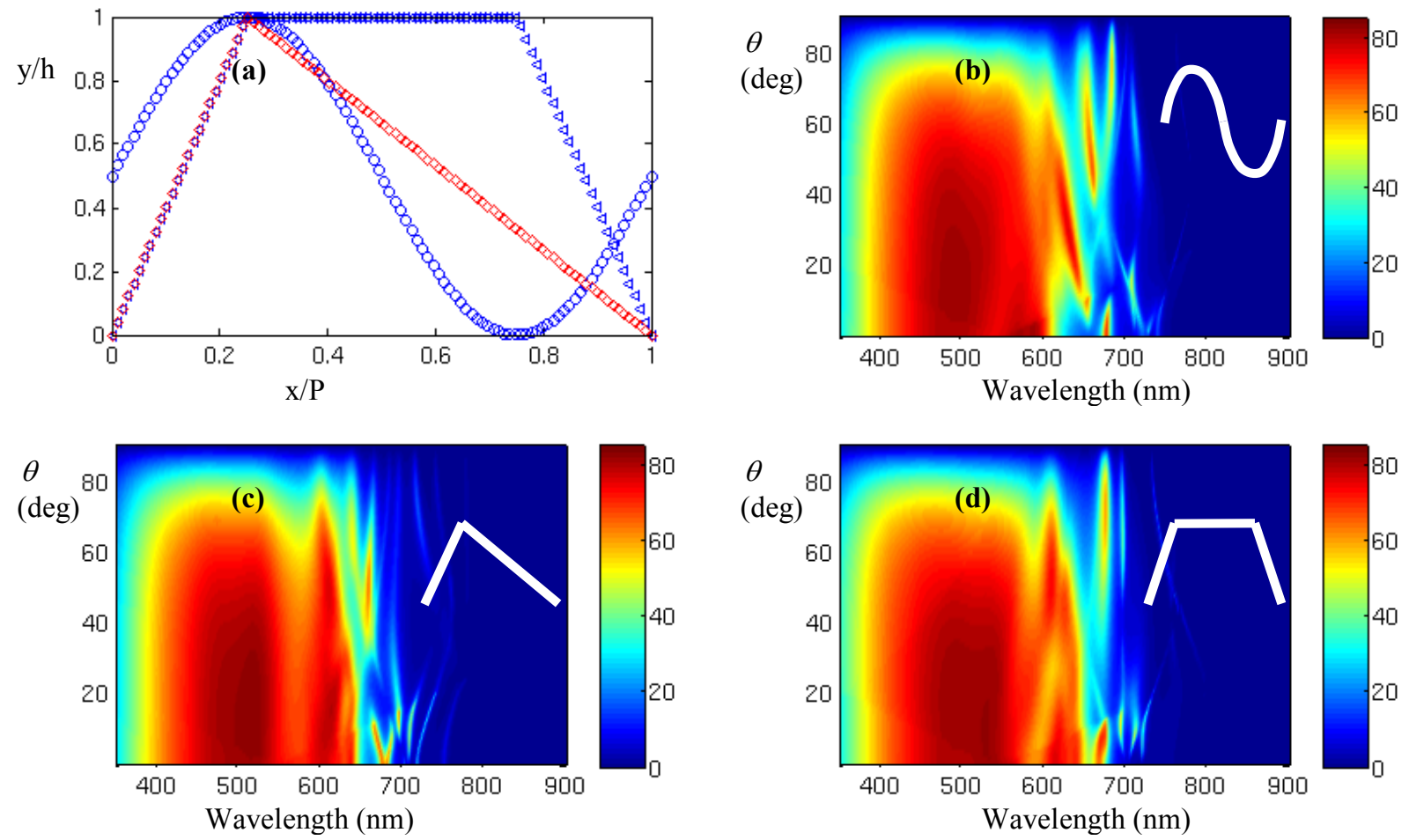

Figure 1 (Color online). (a) Profiles of 1D gratings normalized to grating period $(P)$ and height $(h)$. EQE versus wavelength and incident angle $(\theta)$ for (b) sinusoidal, (c) asymmetric, or (d) symmetric almost binary profile.
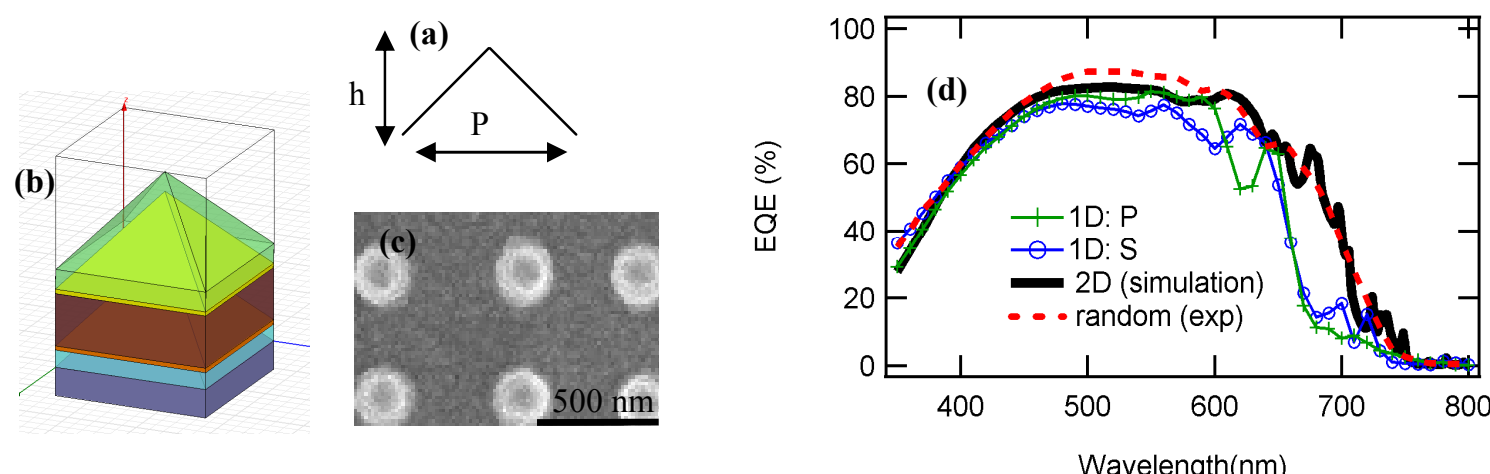

Figure 2 (a) Profile of the 1D grating. (b) Unit cell of the solar cell based on the 2D grating. (c) The top view SEM micrograph of the sample. (d) EQE of cells on 1D and 2D gratings and of the experimental sample.

This work was supported by Swiss National Science Foundation under project number 200020_137700/1.

\section{References}

[1] Z. Yu, A. Raman, and S. Fan, "Fundamental limit of nanophotonic light trapping in solar cells," PNAS 107, 17491-17496 (2010).

[2] V. Ferry, L. Sweatlock, D. Pacifici, and H. Atwater, "Plasmonic nanostructure design for efficient light coupling into solar cells," Nano Lett. 8, 4391-4397 (2008).

[3] X. Sheng, S. G. Johnson, J. Michel, and L. C. Kimerling, "Optimization-based design of surface textures for thin-film Si solar cells," Opt. Express 19, A841-A850 (2011).

[4] C. Battaglia, C. M. Hsu, K. Söderström, J. Escarré, F. J. Haug, M. Charrière, M. Boccard, M. Despeisse, D. Alexander, M. Cantoni, Y. Cui and C. Ballif "Light trapping in solar cells: Can periodic beat random?," ACS Nano 6, 2790-2797 (2012).

[5] S. E. Han and G. Chen, "Toward the Lambertian limit of light trapping in thin nanostructured silicon solar cells," Nano Lett. 10, $4692-4696$ (2010).

[6] C. Heine and R. Morf, "Submicrometer gratings for solar energy applications," Appl. Opt. 34, 2476-2482 (1995). 